\title{
On the Limit Cycles for Continuous and Discontinuous Cubic Differential Systems
}

\author{
Ziguo Jiang $^{1,2}$ \\ ${ }^{1}$ Department of Mathematics and Finances, Aba Teachers University, Wenchuan, Sichuan 623002, China \\ ${ }^{2}$ Institute of Mathematics, Aba Teachers University, Wenchuan, Sichuan 623002, China \\ Correspondence should be addressed to Ziguo Jiang; jzgnl@163.com
}

Received 24 March 2016; Accepted 26 July 2016

Academic Editor: Yuriy V. Rogovchenko

Copyright (c) 2016 Ziguo Jiang. This is an open access article distributed under the Creative Commons Attribution License, which permits unrestricted use, distribution, and reproduction in any medium, provided the original work is properly cited.

\begin{abstract}
We study the number of limit cycles for the quadratic polynomial differential systems $\dot{x}=-y+x^{2}, \dot{y}=x+x y$ having an isochronous center with continuous and discontinuous cubic polynomial perturbations. Using the averaging theory of first order, we obtain that 3 limit cycles bifurcate from the periodic orbits of the isochronous center with continuous perturbations and at least 7 limit cycles bifurcate from the periodic orbits of the isochronous center with discontinuous perturbations. Moreover, this work shows that the discontinuous systems have at least 4 more limit cycles surrounding the origin than the continuous ones.
\end{abstract}

\section{Introduction}

In 1900, Hilbert [1] proposed 23 famous mathematical problems in the second International Congress of Mathematicians, and the second part of the sixteenth problem asks for the maximum $H(n)$ of the number of limit cycles and the relative positions for all planar polynomial differential systems of degree $n$. Mathematicians have done a lot of effective works to research on the numbers of limit cycles for the continuous polynomial system (especially the quadratic polynomial system); see for instance the books $[2,3]$ and the hundreds of references quoted therein. One of the methods for studying on the number of the limit cycles is averaging method. In [4], Buică and Llibre introduced the averaging method for finding limit cycles of continuous differential systems via Brouwer degree. Further, in [5], Llibre et al. used the averaging method for studying the periodic orbits of discontinuous differential systems. Since most phenomena in real life are discontinuous, this created a great interest for the mathematicians to study the limit cycle of discontinuous differential system, especially for the numbers of limit cycles of the discontinuous quadratic polynomial system; see, for instance, [5-9]. For the discontinuous Liénard equations, in [10], Martins and Mereu showed that for any $n \geq 1$ there are differential equations of the form $\ddot{x}+f(x) \dot{x}+\operatorname{sgn}(\dot{x}) g(x)=0$, with $f$ and $g$ being polynomials of degree $n$ and 1, respectively, having $[n / 2]+1$ limit cycles. In [11], Libre and Teixeira provided lower bounds for the maximum number of limit cycles for $m$-piecewise discontinuous polynomial differential equations. For the switching systems, Han and Sheng [12] discussed the bifurcation of limit cycles in piecewise smooth systems via Melnikov functions. Ten limit cycles are found around a center in switching quadratic systems in [13].

Consider a quadratic polynomial system:

$$
\begin{aligned}
& \dot{x}=-y+x^{2}, \\
& \dot{y}=x+x y .
\end{aligned}
$$

Chicone and Jacobs proved in [14] that at most 2 limit cycles bifurcate from the periodic orbits of the isochronous center of system (1). Their study is based on the displacement function using some results of Bautin [15]. In [4], Buică and Llibre easily reproved, using the averaging method, by continuous quadratic polynomial perturbing system (1), the existence of at least 2 limit cycles bifurcating from the periodic orbit of the center of system (1) when this is perturbed inside the class of all quadratic polynomial differential systems. Recently, Llibre and Mereu [7] proved, using the averaging method, by discontinuous quadratic polynomial perturbing system (1), the existence of at least 5 limit cycles bifurcating 
from the periodic orbit of the center of system (1) when this is perturbed inside the class of all quadratic polynomial differential systems, and the discontinuous systems have at least 3 more limit cycles surrounding the origin than the continuous systems.

In this paper, consider the following systems:

$$
\begin{aligned}
& \left(\begin{array}{l}
\dot{x} \\
\dot{y}
\end{array}\right)=\left(\begin{array}{c}
-y+x^{2}+\varepsilon p_{1}(x, y) \\
x+x y+\varepsilon q_{1}(x, y)
\end{array}\right), \\
& \left(\begin{array}{l}
\dot{x} \\
\dot{y}
\end{array}\right)= \begin{cases}\left(\begin{array}{c}
-y+x^{2}+\varepsilon p_{1}(x, y) \\
x+x y+\varepsilon q_{1}(x, y)
\end{array}\right), & y>0, \\
\left(\begin{array}{c}
-y+x^{2}+\varepsilon p_{2}(x, y) \\
x+x y+\varepsilon q_{2}(x, y)
\end{array}\right), & y<0,\end{cases}
\end{aligned}
$$

where $\varepsilon$ is a small parameter, and

$$
\begin{aligned}
p_{1}(x, y)= & a_{1} x+a_{2} y+a_{3} x^{2}+a_{4} x y+a_{5} y^{2}+a_{6} x^{3} \\
& +a_{7} x^{2} y+a_{8} x y^{2}+a_{9} y^{3}, \\
q_{1}(x, y)= & b_{1} x+b_{2} y+b_{3} x^{2}+b_{4} x y+b_{5} y^{2}+b_{6} x^{3} \\
& +b_{7} x^{2} y+b_{8} x y^{2}+b_{9} y^{3}, \\
p_{2}(x, y)= & c_{1} x+c_{2} y+c_{3} x^{2}+c_{4} x y+c_{5} y^{2}+c_{6} x^{3} \\
& +c_{7} x^{2} y+c_{8} x y^{2}+c_{9} y^{3}, \\
q_{2}(x, y)= & d_{1} x+d_{2} y+d_{3} x^{2}+d_{4} x y+d_{5} y^{2}+d_{6} x^{3} \\
& +d_{7} x^{2} y+d_{8} x y^{2}+d_{9} y^{3} .
\end{aligned}
$$

Using averaging method and some results established in [12], we obtain our main results as follows.

Theorem 1. For $|\varepsilon| \neq 0$ sufficiently small there are continuous cubic polynomial differential systems (2) having exactly 3 limit cycles bifurcating from the periodic orbits of isochronous center (1).

Theorem 2. For $|\varepsilon| \neq 0$ sufficiently small there are discontinuous cubic polynomial differential systems (3) having at least 7 limit cycles bifurcating from the periodic orbits of isochronous center (1).

By Theorems 1 and 2, we can get the following.

Corollary 3. Using the averaging method of first order, the discontinuous systems have at least 4 more limit cycles surrounding the origin than the continuous systems when we perturbed the center (1).

In some sense, we extend the work by Llibre and Mereu with the difference of number of limit cycles of discontinuous and continuous differential systems.

\section{Preliminary Results}

In this section, we introduce some preliminary results on the averaging theory which will be applied to study the cubic continuous and discontinuous polynomial systems (2) and (3).

The following theorems are the first-order averaging theory for continuous and discontinuous differential systems. For the proof, we refer the reader to $[4,5]$.

Theorem 4 (see [4]). Consider the following differential system:

$$
x^{\prime}(t)=\varepsilon F(t, x)+\varepsilon^{2} G(t, x, \varepsilon),
$$

where $F: \mathbb{R} \times D \rightarrow \mathbb{R}^{n}, G: \mathbb{R} \times D \times\left(-\varepsilon_{f}, \varepsilon_{f}\right) \rightarrow \mathbb{R}^{n}$ are continuous functions, $T$-periodic in the first variable, and $D$ is an open subset of $\mathbb{R}^{n}$. Define $f: D \rightarrow \mathbb{R}^{n}$ :

$$
f(x)=\frac{1}{T} \int_{0}^{T} F(t, x) d t
$$

and assume that

(i) F and $G$ are locally Lipschitz with respect to $x$.

(ii) For $a \in D$ with $f(a)=0$, there exists a neighborhood $V$ of a such that $f(z) \neq 0$ for all $z \in \bar{V} \backslash\{a\}$ and $d_{B}(f, V, a) \neq 0$.

Then, for $|\varepsilon|>0$ sufficiently small, there exists a T-periodic solution $x(\cdot, \varepsilon)$ of system (5) such that $x(\cdot, \varepsilon) \rightarrow a$ as $\varepsilon \rightarrow 0$.

Theorem 5 (see [5]). Consider the following discontinuous differential system:

$$
x^{\prime}(t)=\varepsilon F(t, x)+\varepsilon^{2} G(t, x, \varepsilon)
$$

with

$$
\begin{aligned}
& F(t, x)=F_{1}(t, x)+\operatorname{sign}(h(t, x)) F_{2}(t, x), \\
& G(t, x)=G_{1}(t, x)+\operatorname{sign}(h(t, x)) G_{2}(t, x),
\end{aligned}
$$

where $F_{1}, F_{2}: \mathbb{R} \times D \rightarrow \mathbb{R}^{n}, G_{1}, G_{2}: \mathbb{R} \times D \times\left(-\varepsilon_{f}, \varepsilon_{f}\right) \rightarrow \mathbb{R}^{n}$ are continuous functions, T-periodic in the first variable, and $D$ is an open subset of $\mathbb{R}^{n}$. We also suppose that $h$ is a $C^{1}$ function having 0 as a regular value. Denote by $\mathscr{M}=h^{-1}(0), \Sigma=0 \times D \nsubseteq$ $\mathscr{M}, \Sigma_{0}=\Sigma \backslash \mathscr{M} \neq \varnothing$, and its elements by $z \equiv(0, z) \notin \mathscr{M}$.

Define the averaged function $f: D \rightarrow \mathbb{R}^{n}$ :

$$
f(x)=\frac{1}{T} \int_{0}^{T} F(t, x) d t .
$$

Assume the following three conditions:

(i) $F_{1}, F_{2}, G_{1}, G_{2}$, and h are locally L-Lipschitz with respect to $x$.

(ii) For $a \in \Sigma_{0}$ with $f(a)=0$, there exists a neighborhood $V$ of a such that $f(z) \neq 0$ for all $z \in \bar{V} \backslash\{a\}$ and $d_{B}(f, V, a) \neq 0$ (i.e., the Brouwer degree of $f$ at $a$ is not zero). 
(iii) If $\partial_{t} h\left(t_{0}, z_{0}\right)=0$ for some $\left(t_{0}, z_{0}\right) \in \mathscr{M}$, then $\left(\left\langle\nabla_{x} h, F_{1}\right\rangle^{2}-\left\langle\nabla_{x} h, F_{2}\right\rangle^{2}\right)\left(t_{0}, z_{0}\right)>0$.

Then, for $|\varepsilon|>0$ sufficiently small, there exists a T-periodic solution $x(\cdot, \varepsilon)$ of system (7) such that $x(\cdot, \varepsilon) \rightarrow$ a as $\varepsilon \rightarrow 0$.

Remark 6 (see [4]). Let $g: D \rightarrow \mathbb{R}^{n}$ be a $C^{1}$ function, with $g(a)=0$, where $D$ is an open subset of $\mathbb{R}^{n}$ and $a \in D$. Whenever $J_{g}(a) \neq 0$, there exists a neighborhood $V$ of a such that $g(z) \neq 0$ for all $z \in \bar{V} \backslash\{a\}$. Then $d_{B}(g, V, a) \neq 0$.

Consider a integrable planar differential system:

$$
\begin{aligned}
& \dot{x}=P(x, y), \\
& \dot{y}=Q(x, y),
\end{aligned}
$$

where $P, Q: \mathbb{R}^{2} \rightarrow \mathbb{R}$ are continuous functions under the assumption

(A1) System (10) has a period annulus around the singular point $(0,0)$ :

$$
\Gamma_{h}:\left\{(x, y) \in \mathbb{R}^{2}: H(x, y)=h, h_{c}<h<h_{s}\right\},
$$

where $H$ is a first integral of (10), $h_{c}$ is the critical level of $H$ corresponding to the center $(0,0)$, and $h_{s}$ denotes the value of $H$ for which the period annulus terminates at a separatrix polycycle. Without loss of generality we can assume that $h_{s}>$ $h_{c} \geq 0$. We denote by $\mu=\mu(x, y)$ the integrating factor of system (10) corresponding to the first integral $H$.

Perturbed systems (10) are as follows:

$$
\begin{aligned}
& \dot{x}=P(x, y)+\varepsilon p(x, y), \\
& \dot{y}=Q(x, y)+\varepsilon q(x, y),
\end{aligned}
$$

where $p, q: \mathbb{R}^{2} \rightarrow \mathbb{R}$ are continuous functions.

In order to apply the averaging method for studying limit cycles of (12) for $\varepsilon$ sufficiently small, we need write system (12) into the standard forms (5) and (7). The following result by [4] provides a way to fulfill it.

Theorem 7 (see [4]). Consider system (10) and its first integral $H$. Assume that (A1) holds for system (10) and that $x Q(x, y)-$ $y P(x, y) \neq 0$ for all $(x, y)$ in the period annulus formed by the ovals $\left\{\Gamma_{h}\right\}$. Let $\rho:\left(\sqrt{h_{c}}, \sqrt{h_{s}}\right) \times[0,2 \pi) \rightarrow[0,+\infty)$ be a continuous function such that

$$
H(\rho(R, \varphi) \cos \varphi, \rho(R, \varphi) \sin \varphi)=R^{2}
$$

for all $R \in\left(\sqrt{h_{c}}, \sqrt{h_{s}}\right)$ and $\varphi \in[0,2 \pi)$. Then the differential equation which describes the dependence between the square root of energy, $R=\sqrt{h}$, and the angle $\varphi$ for system (12) is

$$
\frac{d R}{d \varphi}=\varepsilon \frac{\mu\left(x^{2}+y^{2}\right)(Q p-P q)}{2 R(Q x-P y)+2 R \varepsilon(q x-p y)},
$$

where $x=\rho(R, \varphi) \cos \varphi, y=\rho(R, \varphi) \sin \varphi$.
Remark 8. Further, system (14) can become

$$
\frac{d R}{d \varphi}=\varepsilon F(\varphi, R)+\varepsilon^{2} G(t, x, \varepsilon),
$$

where $F(\varphi, R)=\mu\left(x^{2}+y^{2}\right)(Q p-P q) / 2 R(Q x-P y)$ and $G(t, x, \varepsilon)=\mu\left(x^{2}+y^{2}\right)(Q p-P q) 2 R(p y-q x) / 2 R(Q x-$ $P y)[2 R(Q x-P y)+2 R \varepsilon(q x-p y)]$.

The following lemma presents the version of the formula of the first-order Melnikov function associated with system (12) in the polar coordinates [4].

Lemma 9 (see [4]). Under the conditions of Theorem 7, define

$$
\begin{aligned}
& d(R, \varepsilon)=\varepsilon \int_{0}^{2 \pi} \frac{\mu\left(x^{2}+y^{2}\right)(Q p-P q)}{2 R(Q x-P y)+2 R \varepsilon(q x-p y)} d \varphi, \\
& M_{1}(R)=\int_{0}^{2 \pi} \frac{\mu\left(x^{2}+y^{2}\right)(Q p-P q)}{2 R(Q x-P y)} d \varphi,
\end{aligned}
$$

for system (12), where $\mu=\mu(x, y)$ is the integrating factor of system (10) corresponding to the first integral $H$ and $x=$ $\rho(R, \varphi) \cos \varphi ; y=\rho(R, \varphi) \sin \varphi$. Then $d(R, \varepsilon)$ and $M_{1}(R)$ expressed by (16) are the displacement function and the firstorder Melnikov function of system (12), respectively.

Under the assumption (A1), the assumptions (I) and (II) hold as in [12]. So, we can establish the function $M(h)$ as follows:

$$
\begin{aligned}
M\left(R^{2}\right)=2 \int_{\Gamma_{R^{2}}} q(x, y) d x- & p(x, y) d y, \\
R & =\sqrt{h} \in\left(\sqrt{h_{c}}, \sqrt{h_{s}}\right),
\end{aligned}
$$

since $\int_{\Gamma_{R^{2}}} q(x, y) d x-p(x, y) d y$ is the first-order Melnikov function of system (12). Based on Theorems 4 and 7, Lemma 9, Theorem 1.1 in [12], and (18), we have the following.

Lemma 10. Under the assumption (A1), let $f(R)$ be the averaged function of system (14); then the following relation holds:

$$
4 \pi f(R)=M\left(R^{2}\right)
$$

where $f(R)$ is defined by (6) and $M\left(R^{2}\right)$ is defined by (18).

In order to study the number of zeros of the averaged functions (6) and (9), we will use the following result proved in [16].

Let $A$ be a set and let $f_{1}, f_{2}, \ldots, f_{n}: A \rightarrow \mathbb{R}$. We say that $f_{1}, f_{2}, \ldots, f_{n}$ are linearly independent functions if and only if we have that

$$
\sum_{i=1}^{n} \alpha_{i} f_{i}(a)=0, \quad \forall a \in A \Longrightarrow \alpha_{1}=\alpha_{2}=\cdots=\alpha_{n}=0 .
$$


Proposition 11 (see [16]). If $f_{1}, f_{2}, \ldots, f_{n}: A \rightarrow \mathbb{R}$ are linearly independent, then there exist $a_{1}, a_{2}, \ldots, a_{n-1} \in A$ and $\alpha_{1}, \alpha_{2}, \ldots, \alpha_{n} \in \mathbb{R}$ such that for every $i \in\{1,2, \ldots, n-1\}$

$$
\sum_{k=1}^{n} \alpha_{k} f_{k}\left(a_{i}\right)=0
$$

\section{Proof of Theorem 1}

In this section, we will prove Theorem 1 by using Theorem 4 for the continuous case. We recall that the period annulus of a center is the topological annulus formed by all the periodic orbits surrounding the center which is the only singular point of the system.

A first integral $H$ and an integrating factor $\mu$ in the period annulus of the center of the quadratic differential system (1) have the expressions $H(x, y)=\left(x^{2}+y^{2}\right) /(1+y)^{2}$ and $\mu(x, y)=2 /(1+y)^{3}$, respectively. For this system we note that $h_{c}=0, h_{s}=1$. By (13), the function $\rho$ satisfying the hypotheses of Theorem 7 is given by $\rho(R, \varphi)=R /(1-R \sin \varphi)$, $0<R<1, \varphi \in[0,2 \pi)$. It is easy to know that assumption (A1) holds.

Using Theorem 7, we transform system (2) into the form

$$
\begin{aligned}
& \frac{\frac{\mathrm{d} R}{\mathrm{~d} \varphi}}{=} \\
& \quad \varepsilon \frac{A(\varphi ; a, b) R+B(\varphi ; a, b) R^{2}+C(\varphi ; a, b) R^{3}+D(\varphi ; a, b) R^{4}}{(1-R \sin (\varphi))^{2}} \\
& \quad+\mathcal{O}\left(\varepsilon^{2}\right),
\end{aligned}
$$

where

$$
\begin{aligned}
& A(\varphi ; a, b) \\
& \quad=a_{1} \cos ^{2} \varphi+\left(a_{2}+b_{1}\right) \sin \varphi \cos \varphi+b_{2} \sin ^{2} \varphi
\end{aligned}
$$

$$
\begin{aligned}
B(\varphi ; a, b) & \\
= & \left(a_{4}-b_{1}\right) \cos ^{3} \varphi-\left(2 a_{1}-a_{3}+b_{2}-b_{4}\right) \sin \varphi \cos ^{2} \varphi \\
& -\left(2 a_{2}-a_{5}+3 b_{1}-b_{3}\right) \cos \varphi \sin ^{2} \varphi \\
& +\left(b_{5}-3 b_{2}\right) \sin ^{3} \varphi, \\
C(\varphi ; & ;, b) \\
= & \left(a_{6}-b_{4}\right) \cos ^{4} \varphi \\
& -\left(a_{4}-a_{7}-2 b_{1}+b_{3}-b_{6}\right) \cos ^{3} \varphi \sin \varphi \\
& +\left(a_{1}-a_{3}+a_{8}+2 b_{2}-2 b_{4}-b_{5}+b_{7}\right) \cos ^{2} \varphi \sin ^{2} \varphi \\
& +\left(a_{2}-a_{5}+a_{9}+3 b_{1}-2 b_{3}+b_{8}\right) \cos ^{2} \varphi \sin ^{3} \varphi \\
& +\left(3 b_{2}-2 b_{5}+b_{9}\right) \sin ^{4} \varphi, \\
D( & ; a, b) \\
= & -b_{6} \cos ^{5} \varphi+\left(b_{4}-b_{7}\right) \cos ^{4} \varphi \sin \varphi \\
& -\left(b_{1}-b_{3}+b_{6}+b_{8}\right) \cos ^{3} \varphi \sin ^{2} \varphi \\
& -\left(b_{2}-b_{4}-b_{5}+b_{7}+b_{9}\right) \cos ^{2} \varphi \sin ^{3} \varphi \\
& -\left(b_{1}-b_{3}+b_{8}\right) \cos _{9} \varphi \sin ^{4} \varphi \\
& -\left(\sin ^{5} \varphi,\right. \\
& \\
& \\
& \\
&
\end{aligned}
$$

and $a=\left(a_{1}, a_{2}, \ldots, a_{9}\right), b=\left(b_{1}, b_{2}, \ldots, b_{9}\right)$.

The continuous differential system (22) is under the assumptions of Theorem 4 . So we just need to study the zeros of the averaged function $f:(0,1) \rightarrow \mathbb{R}:$

$$
f(R)=\frac{1}{2 \pi} \int_{0}^{2 \pi} \frac{A(\varphi ; a, b) R+B(\varphi ; a, b) R^{2}+C(\varphi ; a, b) R^{3}+D(\varphi ; a, b) R^{4}}{2(1-R \sin \varphi)^{2}} \mathrm{~d} \varphi .
$$

Using Mathematica (Mathematica software), we compute the above integral and obtain

$$
\begin{aligned}
& f(R)=\frac{1}{2}\left[\left(a_{1}+b_{2}\right) g_{1}+a_{3} g_{2}+a_{6} g_{3}+a_{8} g_{4}+b_{4} g_{5}\right. \\
& \left.\quad+b_{5} g_{6}+b_{7} g_{7}+b_{9} g_{8}\right],
\end{aligned}
$$

where

$$
\begin{aligned}
& g_{1}=R, \\
& g_{2}=\frac{2\left(\sqrt{1-R^{2}}-1\right)-R^{2}\left(\sqrt{1-R^{2}}-2\right)}{R \sqrt{1-R^{2}}}, \\
& g_{3}=\frac{6\left(\sqrt{1-R^{2}}-1\right)-3 R^{2}\left(\sqrt{1-R^{2}}-2\right)}{R \sqrt{1-R^{2}}},
\end{aligned}
$$


We have the equalities

$$
\begin{aligned}
& g_{3}=3 g_{2}, \\
& g_{6}=-g_{2}, \\
& g_{7}=-2 g_{2}-g_{5}, \\
& g_{8}=3 g_{2} .
\end{aligned}
$$

Thus the function $f$ can be written as

$$
\begin{aligned}
f(R) & =\frac{1}{2}\left[\left(a_{1}+b_{2}\right) g_{1}\right. \\
& +\left(a_{3}+3 a_{6}-b_{5}-2 b_{7}+3 b_{9}\right) g_{2}+a_{8} g_{4} \\
& \left.+\left(b_{4}-b_{7}\right) g_{5}\right] .
\end{aligned}
$$

The Wronskian of the functions $g_{1}, g_{2}, g_{4}, g_{5}$ in variable $R$ is

$$
\begin{aligned}
& W\left[g_{1}, g_{2}, g_{4}, g_{5}\right](R) \\
& =\frac{96 \pi^{4} R^{2}\left(R^{4}+4\left(\sqrt{1-R^{2}}-2\right) R^{2}-8 \sqrt{1-R^{2}}+8\right)}{\left(R^{2}-1\right)^{5}} .
\end{aligned}
$$

We have $W\left[g_{1}, g_{2}, g_{4}, g_{5}\right](R) \neq 0$ for all $R \in(0,1)$. In fact, if there exist $R_{0} \in(0,1)$ such that $W\left[g_{1}, g_{2}, g_{4}, g_{5}\right]\left(R_{0}\right)=0$, then

$$
R_{0}^{4}-8 \sqrt{1-R_{0}^{2}}+8=4 R_{0}^{2}\left(2-\sqrt{1-R_{0}^{2}}\right)
$$

and it is not difficult to obtain that

$$
-R_{0}^{8}=0
$$

Obviously, it is impossible for all $R_{0} \in(0,1)$. Then, the functions $g_{1}, g_{2}, g_{4}, g_{5}$ in $R$ are linearly independent. By Proposition 11, there exist $\alpha_{i} \in \mathbb{R}, i=1,2,3,4$, such that the linear combination $\alpha_{1} g_{1}+\alpha_{2} g_{2}+\alpha_{3} g_{4}+\alpha_{4} g_{5}$ of four functions $g_{1}, g_{2}, g_{4}, g_{5}$ has at least 3 zeros $R_{1}, R_{2}, R_{3}$; that is, for all $i=1,2,3$, the following equations hold:

$$
\alpha_{1} g_{1}\left(R_{i}\right)+\alpha_{2} g_{2}\left(R_{i}\right)+\alpha_{3} g_{4}\left(R_{i}\right)+\alpha_{4} g_{5}\left(R_{i}\right)=0 \text {. }
$$

Comparing (28) and (32), we get a set of equations about variables $a_{1}, a_{3}, a_{6}, a_{8}, b_{4}, b_{5}, b_{7}, b_{9}$ :

$$
\begin{aligned}
& \alpha_{1}=a_{1}+b_{2}, \\
& \alpha_{2}=a_{3}+3 a_{6}-b_{5}-2 b_{7}+3 b_{9}, \\
& \alpha_{3}=a_{8}, \\
& \alpha_{4}=b_{4}-b_{7} .
\end{aligned}
$$

The rank of coefficients matrix of (33) is 4; then the solutions of these equations exist. Thus there exist $a_{j}, b_{j} \in \mathbb{R}$, $j=1,2, \ldots, 9$ such that the averaged function $f(R)$ has at least 3 zeros $R_{1}, R_{2}, R_{3}$, where $a_{1}, a_{3}, a_{6}, a_{8}, b_{4}, b_{5}, b_{7}, b_{9}$ are the solutions of (33); other ones are 0.
On the other hand, let $R=\left(1-w^{2}\right) /\left(1+w^{2}\right), 0<w<1$; the averaged function $f(R)$ becomes

$$
f(R)=\frac{(1-w)}{2 w(1+w)\left(1+w^{2}\right)^{2}} g(w),
$$

where

$$
\begin{aligned}
g(w)= & N_{1}+N_{2} w+N_{3} w^{2}+N_{4} w^{3}+N_{3} w^{4}+N_{2} w^{5} \\
& +N_{1} w^{6} \\
N_{1}= & a_{8}, \\
N_{2}= & a_{1}+a_{3}+3 a_{6}-3 a_{8}+b_{2}-b_{4}-b_{5}-b_{7}+3 b_{9}, \\
N_{3}= & 2 a_{1}-2 a_{3}-6 a_{6}+5 a_{8}+2 b_{2}-2 b_{4}+2 b_{5}+6 b_{7} \\
& -6 b_{9}, \\
N_{4}= & 2 a_{1}+2 a_{3}+6 a_{6}-6 a_{8}+2 b_{2}+6 b_{4}-2 b_{5} \\
& -10 b_{7}+6 b_{9} .
\end{aligned}
$$

As a result of the symmetry of coefficients of $g(w)$, we know that if $w_{0} \neq 0$ is one root of $g(w)=0$, so is $1 / w_{0}$, but only one of $w_{0}$ and $1 / w_{0}$ is in interval $(0,1)$. Hence, the fact that $g(w)$ has at most 3 zeros in $w \in(0,1)$ implies that there exist at most 3 zeros for $f(R)$ in $R \in(0,1)$.

Thus, the averaged function $f(R)$ has exactly 3 zeros. By Theorem 4, Lemmas 9 and 10, and Theorem 1.1 in [12], we get that there are exactly 3 limit cycles bifurcate from the period annulus around the isochronous center for (2) with sufficiently small $|\varepsilon|$. This completes the proof of Theorem 1 .

\section{Example}

In this section, we not only provide some examples satisfying the property of Theorem 1, but also introduce a method to construct such systems.

Suppose that

$$
\begin{gathered}
\tilde{g}(w)=\left(w-\frac{1}{10}\right)\left(w-\frac{1}{5}\right)\left(w-\frac{1}{2}\right)(w-10)(w-5) \\
\cdot(w-2)=1-\frac{89}{5} w+\frac{9377}{100} w^{2}-\frac{1669}{10} w^{3} \\
+\frac{9377}{100} w^{4}-\frac{89}{5} w^{5}+w^{6}
\end{gathered}
$$

Take the constants

$$
\begin{aligned}
& N_{1}=1, \\
& N_{2}=-\frac{89}{5}, \\
& N_{3}=\frac{9377}{100}, \\
& N_{4}=-\frac{1669}{10} .
\end{aligned}
$$


From (35) and (37), we have

$$
\begin{aligned}
& a_{1}=-\frac{81}{50}-b_{2}, \\
& a_{3}=-\frac{11837}{400}-3 a_{6}+b_{5}+2 b_{7}-3 b_{9},
\end{aligned}
$$

$$
\begin{aligned}
& a_{8}=1, \\
& b_{4}=-\frac{1313}{80}+b_{7},
\end{aligned}
$$

where $a_{6}, b_{2}, b_{5}, b_{7}, b_{9}$ and other ones are any real constants.

Hence, in system (2), for the sufficiently small $|\varepsilon|$, we obtain a family of systems:

$$
\begin{aligned}
& \left(\begin{array}{l}
\dot{x} \\
\dot{y}
\end{array}\right) \\
& =\left(\begin{array}{c}
-y+x^{2}+\varepsilon\left[\left(-\frac{81}{50}-b_{2}\right) x+a_{2} y+\left(-\frac{11837}{400}-3 a_{6}+b_{5}+2 b_{7}-3 b_{9}\right) x^{2}+a_{4} x y+a_{5} y^{2}+a_{6} x^{3}+a_{7} x^{2} y+x y^{2}+a_{9} y^{3}\right] \\
x+x y+\varepsilon\left[b_{1} x+b_{2} y+b_{3} x^{2}+\left(-\frac{1313}{80}+b_{7}\right) x y+b_{5} y^{2}+b_{6} x^{3}+b_{7} x^{2} y+b_{8} x y^{2}+b_{9} y^{3}\right]
\end{array}\right) .
\end{aligned}
$$

The averaged function of systems (39) is

$$
\begin{aligned}
f(R)= & \frac{(1-w)}{2 w(1+w)\left(1+w^{2}\right)^{2}}\left(w-\frac{1}{10}\right)\left(w-\frac{1}{5}\right) \\
& \cdot\left(w-\frac{1}{2}\right)(w-10)(w-5)(w-2) .
\end{aligned}
$$

Apparently, $f(R)$ has exactly 3 positive zeros in $(0,1)$, denoted by

$$
\begin{aligned}
& R_{1}=\frac{99}{101}, \\
& R_{2}=\frac{12}{13}, \\
& R_{3}=\frac{3}{5},
\end{aligned}
$$

corresponding to $w_{1}=1 / 10, w_{2}=1 / 5$, and $w_{3}=12$ in $R \in(0,1)$. Moreover, we have

$$
\begin{aligned}
& \frac{d f\left(R_{1}\right)}{d R}=\frac{75411 \pi}{1000}, \\
& \frac{d f\left(R_{2}\right)}{d R}=-\frac{1323 \pi}{125}, \\
& \frac{d f\left(R_{3}\right)}{d R}=\frac{513 \pi}{200} .
\end{aligned}
$$

That is to say, $f^{\prime}\left(R_{i}\right) \neq 0, i \in\{1,2,3\}$; it follows from Theorem 4 and Lemmas 9 and 10 that, for the sufficiently small $|\varepsilon|$, system (39) has exactly 3 limit cycles emerging from the period annulus of the corresponding unperturbed system.

\section{Proof of Theorem 2}

In this section, we will prove Theorem 2 by using the first-order averaging method for the continuous case. The unperturbed system of system (3) is also system (1); then using Theorem 7 and Remark 8 we transform system (3) into the form

$$
\frac{\mathrm{d} R}{\mathrm{~d} \varphi}= \begin{cases}\varepsilon \frac{A(\varphi ; a, b) R+B(\varphi ; a, b) R^{2}+C(\varphi ; a, b) R^{3}+D(\varphi ; a, b) R^{4}}{2(1-R \sin \varphi)^{2}}+\mathcal{O}\left(\varepsilon^{2}\right), & y>0, \\ \varepsilon \frac{A(\varphi ; c, d) R+B(\varphi ; c, d) R^{2}+C(\varphi ; c, d) R^{3}+D(\varphi ; c, d) R^{4}}{2(1-R \sin \varphi)^{2}}+\mathcal{O}\left(\varepsilon^{2}\right), & y<0,\end{cases}
$$


where

$A(\varphi ; a, b)$

$=a_{1} \cos ^{2} \varphi+\left(a_{2}+b_{1}\right) \sin \varphi \cos \varphi+b_{2} \sin ^{2} \varphi$,

$B(\varphi ; a, b)$

$=\left(a_{4}-b_{1}\right) \cos ^{3} \varphi-\left(2 a_{1}-a_{3}+b_{2}-b_{4}\right) \sin \varphi \cos ^{2} \varphi$

$-\left(2 a_{2}-a_{5}+3 b_{1}-b_{3}\right) \cos \varphi \sin ^{2} \varphi$

$+\left(b_{5}-3 b_{2}\right) \sin ^{3} \varphi$,

$C(\varphi ; a, b)$

$=\left(a_{6}-b_{4}\right) \cos ^{4} \varphi$

$-\left(a_{4}-a_{7}-2 b_{1}+b_{3}-b_{6}\right) \cos ^{3} \varphi \sin \varphi$

$+\left(a_{1}-a_{3}+a_{8}+2 b_{2}-2 b_{4}-b_{5}+b_{7}\right) \cos ^{2} \varphi \sin ^{2} \varphi$ $+\left(a_{2}-a_{5}+a_{9}+3 b_{1}-2 b_{3}+b_{8}\right) \cos \varphi \sin ^{3} \varphi$

$+\left(3 b_{2}-2 b_{5}+b_{9}\right) \sin ^{4} \varphi$,

$D(\varphi ; a, b)$

$=-b_{6} \cos ^{5} \varphi+\left(b_{4}-b_{7}\right) \cos ^{4} \varphi \sin \varphi$

$-\left(b_{1}-b_{3}+b_{6}+b_{8}\right) \cos ^{3} \varphi \sin ^{2} \varphi$

$-\left(b_{2}-b_{4}-b_{5}+b_{7}+b_{9}\right) \cos ^{2} \varphi \sin ^{3} \varphi$

$-\left(b_{1}-b_{3}+b_{8}\right) \cos \varphi \sin ^{4} \varphi$

$-\left(b_{2}-b_{5}+b_{9}\right) \sin ^{5} \varphi$,

and $a=\left(a_{1}, a_{2}, \ldots, a_{9}\right), b=\left(b_{1}, b_{2}, \ldots, b_{9}\right), c=$ $\left(c_{1}, c_{2}, \ldots, c_{9}\right)$, and $d=\left(d_{1}, d_{2}, \ldots, d_{9}\right)$.

The discontinuous differential system (43) is under the assumptions of Theorem 5. So we just need to study the zeros of the averaged function $f:(0,1) \rightarrow \mathbb{R}$ :

$$
\begin{gathered}
f(R)=\frac{1}{2 \pi}\left[\int_{0}^{\pi} \frac{A(\varphi ; a, b) R+B(\varphi ; a, b) R^{2}+C(\varphi ; a, b) R^{3}+D(\varphi ; a, b) R^{4}}{2(1-R \sin \varphi)^{2}} \mathrm{~d} \varphi\right. \\
\left.+\int_{\pi}^{2 \pi} \frac{A(\varphi ; c, d) R+B(\varphi ; c, d) R^{2}+C(\varphi ; c, d) R^{3}+D(\varphi ; c, d) R^{4}}{2(1-R \sin \varphi)^{2}} \mathrm{~d} \varphi\right] .
\end{gathered}
$$

The above integral is calculated by the mathematical software Mathematica obtaining

$$
\begin{aligned}
& f(R)=\frac{1}{2 \pi}\left[\left(a_{1}+c_{1}\right) g_{1}+a_{3} g_{2}+a_{6} g_{3}+a_{8} g_{4}+b_{2} g_{5}\right. \\
& \quad+b_{4} g_{6}+b_{5} g_{7}+b_{7} g_{8}+b_{9} g_{9}+c_{3} g_{10}+c_{6} g_{11} \\
& \left.\quad+c_{8} g_{12}+d_{2} g_{13}+d_{4} g_{14}+d_{5} g_{15}+d_{7} g_{16}+d_{9} g_{17}\right]
\end{aligned}
$$

where

$$
\begin{aligned}
& g_{1}=\frac{\pi R}{2}, \\
& g_{2} \\
& =\frac{4 \sqrt{1-R^{2}} R+\pi\left(2\left(\sqrt{1-R^{2}}-1\right)-R^{2}\left(\sqrt{1-R^{2}}-2\right)\right)}{2 R \sqrt{1-R^{2}}} \\
& \quad+\frac{2\left(R^{2}-1\right) \arctan \left(R / \sqrt{1-R^{2}}\right)}{R \sqrt{1-R^{2}}},
\end{aligned}
$$

$g_{3}$

$$
\begin{aligned}
= & -2 R^{2}+\frac{3 \pi R}{\sqrt{1-R^{2}}}-\frac{3 \pi}{R \sqrt{1-R^{2}}} \\
& +\frac{6\left(R^{2}-1\right) \arctan \left(R / \sqrt{1-R^{2}}\right)}{R \sqrt{1-R^{2}}}-\frac{3 \pi R}{2}+\frac{3 \pi}{R}+6,
\end{aligned}
$$

$g_{4}$

$$
\begin{aligned}
= & -\frac{2 \pi R}{\sqrt{1-R^{2}}}+\frac{3 \pi}{R \sqrt{1-R^{2}}}-\frac{4 R \arctan \left(R / \sqrt{1-R^{2}}\right)}{\sqrt{1-R^{2}}} \\
& +\frac{6 \arctan \left(R / \sqrt{1-R^{2}}\right)}{R \sqrt{1-R^{2}}}+\frac{\pi R}{2}-\frac{3 \pi}{R}-6,
\end{aligned}
$$

$g_{5}=\frac{\pi R}{2}-2 R^{2}$,

$g_{6}$

$$
\begin{aligned}
= & -2 R^{2}+\frac{2 \pi R}{\sqrt{1-R^{2}}}-\frac{\pi}{R \sqrt{1-R^{2}}} \\
& +\frac{4 R \arctan \left(R / \sqrt{1-R^{2}}\right)}{\sqrt{1-R^{2}}}-\frac{2 \arctan \left(R / \sqrt{1-R^{2}}\right)}{R \sqrt{1-R^{2}}} \\
& -\frac{\pi R^{3}}{\sqrt{1-R^{2}}}-\frac{2 R^{3} \arctan \left(R / \sqrt{1-R^{2}}\right)}{\sqrt{1-R^{2}}}-\frac{3 \pi R}{2}+\frac{\pi}{R} \\
& +2,
\end{aligned}
$$

$g_{7}$

$=2 R^{2}-\frac{\pi R}{\sqrt{1-R^{2}}}+\frac{\pi}{R \sqrt{1-R^{2}}}$ 


$$
\begin{aligned}
& -\frac{2 R \arctan \left(R / \sqrt{1-R^{2}}\right)}{\sqrt{1-R^{2}}}+\frac{2 \arctan \left(R / \sqrt{1-R^{2}}\right)}{R \sqrt{1-R^{2}}} \\
& +\frac{\pi R}{2}-\frac{\pi}{R}-2,
\end{aligned}
$$

$g_{8}$

$$
\begin{aligned}
= & 4 R^{2}-\frac{4 \pi R}{\sqrt{1-R^{2}}}+\frac{3 \pi}{R \sqrt{1-R^{2}}} \\
& -\frac{8 R \arctan \left(R / \sqrt{1-R^{2}}\right)}{\sqrt{1-R^{2}}}+\frac{6 \arctan \left(R / \sqrt{1-R^{2}}\right)}{R \sqrt{1-R^{2}}} \\
& +\frac{\pi R^{3}}{\sqrt{1-R^{2}}}+\frac{2 R^{3} \arctan \left(R / \sqrt{1-R^{2}}\right)}{\sqrt{1-R^{2}}}+\frac{5 \pi R}{2}-\frac{3 \pi}{R} \\
& -6, \\
g_{9} & -2 R^{2}+\frac{3 \pi R}{\sqrt{1-R^{2}}}-\frac{3 \pi}{R \sqrt{1-R^{2}}} \\
& +\frac{6 R \arctan \left(R / \sqrt{1-R^{2}}\right)}{\sqrt{1-R^{2}}}-\frac{6 \arctan \left(R / \sqrt{1-R^{2}}\right)}{R \sqrt{1-R^{2}}} \\
& -\frac{3 \pi R}{2}+\frac{3 \pi}{R}+6,
\end{aligned}
$$

$g_{10}$

$$
\begin{gathered}
=-\frac{4 \sqrt{1-R^{2}} R+\pi\left(\left(\sqrt{1-R^{2}}-2\right) R^{2}-2 \sqrt{1-R^{2}}+2\right)}{2 R \sqrt{1-R^{2}}} \\
+\frac{2 \arctan \left(R / \sqrt{1-R^{2}}\right)}{R \sqrt{1-R^{2}}}-\frac{2 R \arctan \left(R / \sqrt{1-R^{2}}\right)}{\sqrt{1-R^{2}}},
\end{gathered}
$$

$g_{11}$

$$
\begin{aligned}
= & 2 R^{2}+\frac{3 \pi R}{\sqrt{1-R^{2}}}-\frac{3 \pi}{R \sqrt{1-R^{2}}} \\
& -\frac{6\left(R^{2}-1\right) \arctan \left(R / \sqrt{1-R^{2}}\right)}{R \sqrt{1-R^{2}}}-\frac{3 \pi R}{2}+\frac{3 \pi}{R}-6,
\end{aligned}
$$

$g_{12}$

$$
\begin{gathered}
=-\frac{2 \pi R}{\sqrt{1-R^{2}}}+\frac{3 \pi}{R \sqrt{1-R^{2}}}+\frac{4 R \arctan \left(R / \sqrt{1-R^{2}}\right)}{\sqrt{1-R^{2}}} \\
-\frac{6 \arctan \left(R / \sqrt{1-R^{2}}\right)}{R \sqrt{1-R^{2}}}+\frac{\pi R}{2}-\frac{3 \pi}{R}+6,
\end{gathered}
$$$$
g_{13}=2 R^{2}+\frac{\pi R}{2},
$$

$g_{14}$

$$
\begin{aligned}
= & 2 R^{2}+\frac{2 \pi R}{\sqrt{1-R^{2}}}-\frac{\pi}{R \sqrt{1-R^{2}}} \\
& -\frac{4 R \arctan \left(R / \sqrt{1-R^{2}}\right)}{\sqrt{1-R^{2}}}+\frac{2 \arctan \left(R / \sqrt{1-R^{2}}\right)}{R \sqrt{1-R^{2}}}
\end{aligned}
$$

$$
\begin{aligned}
& -\frac{\pi R^{3}}{\sqrt{1-R^{2}}}+\frac{2 R^{3} \arctan \left(R / \sqrt{1-R^{2}}\right)}{\sqrt{1-R^{2}}}-\frac{3 \pi R}{2}+\frac{\pi}{R} \\
& -2,
\end{aligned}
$$

$g_{15}$

$$
\begin{aligned}
= & -2 R^{2}-\frac{\pi R}{\sqrt{1-R^{2}}}+\frac{\pi}{R \sqrt{1-R^{2}}} \\
& +\frac{2 R \arctan \left(R / \sqrt{1-R^{2}}\right)}{\sqrt{1-R^{2}}}-\frac{2 \arctan \left(R / \sqrt{1-R^{2}}\right)}{R \sqrt{1-R^{2}}} \\
& +\frac{\pi R}{2}-\frac{\pi}{R}+2,
\end{aligned}
$$

$g_{16}$

$$
\begin{aligned}
= & -4 R^{2}-\frac{4 \pi R}{\sqrt{1-R^{2}}}+\frac{3 \pi}{R \sqrt{1-R^{2}}} \\
& +\frac{8 R \arctan \left(R / \sqrt{1-R^{2}}\right)}{\sqrt{1-R^{2}}}-\frac{6 \arctan \left(R / \sqrt{1-R^{2}}\right)}{R \sqrt{1-R^{2}}} \\
& +\frac{\pi R^{3}}{\sqrt{1-R^{2}}}-\frac{2 R^{3} \arctan \left(R / \sqrt{1-R^{2}}\right)}{\sqrt{1-R^{2}}}+\frac{5 \pi R}{2}-\frac{3 \pi}{R} \\
& +6
\end{aligned}
$$

$g_{17}$

$$
\begin{aligned}
= & 2 R^{2}+\frac{3 \pi R}{\sqrt{1-R^{2}}}-\frac{3 \pi}{R \sqrt{1-R^{2}}} \\
& -\frac{6 R \arctan \left(R / \sqrt{1-R^{2}}\right)}{\sqrt{1-R^{2}}}+\frac{6 \arctan \left(R / \sqrt{1-R^{2}}\right)}{R \sqrt{1-R^{2}}} \\
& -\frac{3 \pi R}{2}+\frac{3 \pi}{R}-6 .
\end{aligned}
$$

The following equalities hold:

$$
\begin{aligned}
& g_{5}=g_{1}-3 g_{2}+g_{3}, \\
& g_{7}=2 g_{2}-g_{3}, \\
& g_{8}=g_{2}-g_{3}-g_{6}, \\
& g_{9}=g_{3}, \\
& g_{11}=3 g_{2}-g_{3}+3 g_{10}, \\
& g_{13}=g_{1}+3 g_{2}-g_{3}, \\
& g_{15}=-3 g_{2}+g_{3}-g_{10}, \\
& g_{16}=-3 g_{2}+g_{3}-2 g_{10}-g_{14}, \\
& g_{17}=3 g_{2}-g_{3}+3 g_{10} .
\end{aligned}
$$


So we can rewrite the function $f$ as

$$
\begin{aligned}
& f(R)=\frac{1}{2 \pi}\left[\left(a_{1}+b_{2}+c_{1}+d_{2}\right) g_{1}+\left(a_{3}-3 b_{2}+2 b_{5}\right.\right. \\
& \left.+b_{7}+3 c_{6}+3 d_{2}-3 d_{5}-5 d_{7}+3 d_{9}\right) g_{2}+\left(a_{6}+b_{2}\right. \\
& \left.\quad-b_{5}-b_{7}+b_{9}-c_{6}-d_{2}+d_{5}+d_{7}-d_{9}\right) g_{3}+a_{8} g_{4} \\
& \quad+\left(b_{4}-b_{7}\right) g_{6}+\left(c_{3}+3 c_{6}-d_{5}+3 d_{9}\right) g_{10}+c_{8} g_{12} \\
& \left.\quad+\left(d_{4}-d_{7}\right) g_{14}\right] .
\end{aligned}
$$

Through calculation, the Wronskian of the eight functions $g_{i}, i=1,2,3,4,6,10,12,14$ in variable $R$ is valued as $W\left[g_{1}, g_{2}, g_{3}, g_{4}, g_{6}, g_{10}, g_{12}, g_{14}\right](0.1)=4.66942 \neq 0$ at $R=0.1$, so the eight functions are linearly independent. By Proposition 11, there exist $\alpha_{j} \in \mathbb{R}, j=1,2, \ldots, 8$, and at least seven $R_{i} \in(0,1), i=1,2, \ldots, 7$, such that the equality

$$
\begin{gathered}
\alpha_{1} g_{1}\left(R_{i}\right)+\alpha_{2} g_{2}\left(R_{i}\right)+\alpha_{3} g_{3}\left(R_{i}\right)+\alpha_{4} g_{4}\left(R_{i}\right) \\
+\alpha_{5} g_{6}\left(R_{i}\right)+\alpha_{6} g_{10}\left(R_{i}\right)+\alpha_{7} g_{12}\left(R_{i}\right) \\
+\alpha_{8} g_{14}\left(R_{i}\right)=0
\end{gathered}
$$

holds, for all $i \in\{1,2,3,4,5,6,7\}$.

From (49) and (50), we obtain linear equations about variables $a_{1}, a_{3}, a_{6}, a_{8}, b_{2}, b_{4}, b_{5}, b_{7}, b_{9}, c_{1}, c_{3}, c_{6}, c_{8}, d_{2}, d_{4}, d_{5}, d_{7}, d_{9}$, and it is easy to know that these linear equations have solutions. Hence, there exist $a_{j}, b_{j}, c_{j}, d_{j} \in \mathbb{R}, j \in\{1,2$, $3,4,5,6,7,8,9\}$ (where $a_{1}, a_{3}, a_{6}, a_{8}, b_{2}, b_{4}, b_{5}, b_{7}, b_{9}, c_{1}, c_{3}, c_{6}$, $c_{8}, d_{2}, d_{4}, d_{5}, d_{7}, d_{9}$ are the solutions of these equations, and other ones are 0 ), such that the averaged function $f(R)$ has at least 7 zeros $R_{i} \in(0,1), i \in\{1,2,3,4,5,6,7\}$.

In short, there are discontinuous cubic polynomial systems (3) having at least 7 limit cycles bifurcating from the periodic orbits of the isochronous center of system (1) by using Theorem 5. This completes the proof of Theorem 2 .

\section{Example}

In this section, we will introduce a method to construct some examples satisfying the property of Theorem 2; moreover, we will provide such systems.

Firstly, we take $R_{i}=i / 10, i=1,2, \ldots, 7$, in (50), respectively. Thus, we get linear equations about variable $\alpha_{j} \epsilon$ $\mathbb{R}, j=1,2, \ldots, 8$. Solve these equations, and we choose one of the solutions of these equations:

$$
\begin{aligned}
& \alpha_{1}=-0.00217973183257539, \\
& \alpha_{2}=492.047754227194, \\
& \alpha_{3}=-171.09394768524, \\
& \alpha_{4}=1.53874330716414, \\
& \alpha_{5}=-19.6427452695672, \\
& \alpha_{6}=372.273977923452, \\
& \alpha_{7}=-113.700137927805, \\
& \alpha_{8}=100 .
\end{aligned}
$$

From (49) and (50), we obtain the following linear equations about variables $a_{1}, a_{3}, a_{6}, a_{8}, b_{2}, b_{4}, b_{5}, b_{7}, b_{9}, c_{1}, c_{3}, c_{6}, c_{8}$, $d_{2}, d_{4}, d_{5}, d_{7}, d_{9}$ :

$$
\begin{aligned}
& a_{1}+b_{2}+c_{1}+d_{2}=\alpha_{1} \text {, } \\
& a_{3}-3 b_{2}+2 b_{5}+b_{7}+3 c_{6}+3 d_{2}-3 d_{5}-5 d_{7}+3 d_{9} \\
& =\alpha_{2} \text {, } \\
& a_{6}+b_{2}-b_{5}-b_{7}+b_{9}-c_{6}-d_{2}+d_{5}+d_{7}-d_{9}=\alpha_{3} \text {, } \\
& a_{8}=\alpha_{4} \text {, } \\
& b_{4}-b_{7}=\alpha_{5} \text {, } \\
& c_{3}+3 c_{6}-d_{5}+3 d_{9}=\alpha_{6} \text {, } \\
& c_{8}=\alpha_{7}, \\
& d_{4}-d_{7}=\alpha_{8} \text {. }
\end{aligned}
$$

Second, solve (52), in (3), and we choose

$$
\begin{aligned}
a_{1}= & -0.00217973183298289-b_{2}-c_{1}-d_{2}, \\
a_{3}= & 492.047754229033+3 b_{2}-2 b_{5}-b_{7}-3 c_{6}-3 d_{2} \\
& +3 d_{5}+5 d_{7}-3 d_{9}, \\
a_{6}= & -171.093947685661-b_{2}+b_{5}+b_{7}-b_{9}+c_{6} \\
& +d_{2}-d_{5}-d_{7}+d_{9}, \\
a_{8}= & 1.5387433071157, \\
b_{4}= & -19.642745269155+b_{7}, \\
c_{3}= & 372.273977925687-3 c_{6}+d_{5}-3 d_{9}, \\
c_{8}= & -113.700137929543, \\
d_{4}= & 100+d_{7},
\end{aligned}
$$


and all other coefficients are any real constants; then system (3) becomes

$$
\left(\begin{array}{l}
\dot{x} \\
\dot{y}
\end{array}\right)=\left\{\begin{array}{cc}
\left(\begin{array}{cc}
-y+x^{2}+\varepsilon X_{1}(x, y) \\
x+x y+\varepsilon Y_{1}(x, y)
\end{array}\right), & y>0 \\
\left(\begin{array}{c}
-y+x^{2}+\varepsilon X_{2}(x, y) \\
x+x y+\varepsilon Y_{2}(x, y)
\end{array}\right), & y<0
\end{array}\right.
$$

where

$$
\begin{aligned}
& X_{1}(x, y)=\left(-0.00217973183298289-b_{2}-c_{1}-d_{2}\right) \\
& \cdot x+a_{2} y+\left(492.047754229033+3 b_{2}-2 b_{5}-b_{7}\right. \\
& \left.\quad-3 c_{6}-3 d_{2}+3 d_{5}+5 d_{7}-3 d_{9}\right) x^{2}+a_{4} x y+a_{5} y^{2} \\
& +\left(-171.093947685661-b_{2}+b_{5}+b_{7}-b_{9}+c_{6}\right. \\
& \left.\quad+d_{2}-d_{5}-d_{7}+d_{9}\right) x^{3}+a_{7} x^{2} y \\
& \quad+1.5387433071157 x y^{2}+a_{9} y^{3} \\
& Y_{1}(x, y)=b_{1} x+b_{2} y+b_{3} x^{2}+(-19.642745269155 \\
& \left.\quad+b_{7}\right) x y+b_{5} y^{2}+b_{6} x^{3}+b_{7} x^{2} y+b_{8} x y^{2}+b_{9} y^{3} \\
& X_{2}(x, y)=c_{1} x+c_{2} y+\left(372.273977925687-3 c_{6}\right. \\
& \left.\quad+d_{5}-3 d_{9}\right) x^{2}+c_{4} x y+c_{5} y^{2}+c_{6} x^{3}+c_{7} x^{2} y \\
& \quad-113.700137929543 x y^{2}+c_{9} y^{3} \\
& Y_{2}(x, y)=d_{1} x+d_{2} y+d_{3} x^{2}+\left(100+d_{7}\right) x y+d_{5} y^{2} \\
& \quad+d_{6} x^{3}+d_{7} x^{2} y+d_{8} x y^{2}+d_{9} y^{3} .
\end{aligned}
$$

The averaged function of system (3) is

$$
\begin{aligned}
& f(R) \\
& \quad=\frac{1}{2 \pi R \sqrt{1-R^{2}}}\left[R^{2}\left(2312.45-1106.27 \sqrt{1-R^{2}}\right)\right. \\
& \quad-252.45 R^{4}-1717.73 \sqrt{1-R^{2}} R \\
& +2412.37 \sqrt{1-R^{2}} \\
& +\left(239.285 R^{4}-1726.54 R^{2}+1717.73\right) \\
& \quad \arctan \left(\frac{R}{\sqrt{1-R^{2}}}\right)+581.473 \sqrt{1-R^{2}} R^{3} \\
& -2412.37] .
\end{aligned}
$$

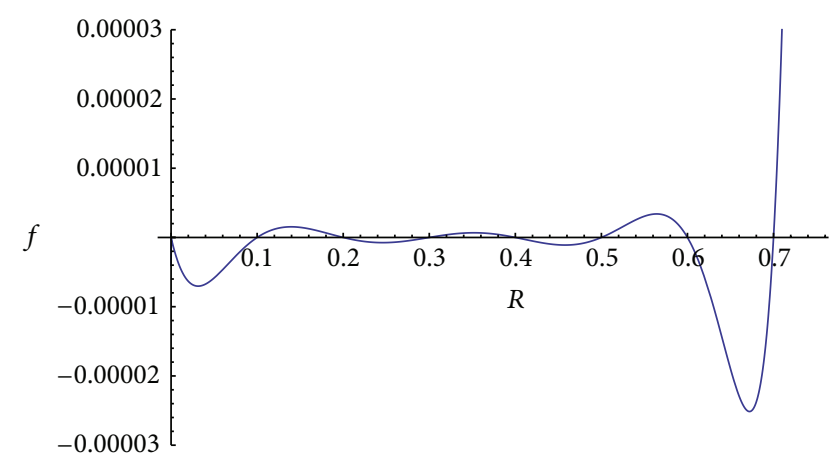

Figure 1

The function $f(R)$ has at lest 7 zeros:

$$
\begin{aligned}
R_{1} & =0.1 \\
R_{2} & =0.2 \\
R_{3} & =0.3 \\
R_{4} & =0.4 \\
R_{5} & =0.5 \\
R_{6} & =0.6 \\
R_{7} & =0.7 \\
f^{\prime}(0.1) & =0.0000825499574205021 \\
f^{\prime}(0.2) & =-0.0000302356277760415 \\
f^{\prime}(0.3) & =0.0000207317994750498 \\
f^{\prime}(0.4) & =-0.0000247975117812018 \\
f^{\prime}(0.5) & =0.0000522877306220519 \\
f^{\prime}(0.6) & =-0.000213685595035765 \\
f^{\prime}(0.7) & =0.00226678597503152
\end{aligned}
$$

that is, $f^{\prime}\left(R_{i}\right) \neq 0, i=1,2, \ldots, 7 . f(R)$ is shown in Figure 1 . Hence, by Theorem 5 , it follows that, for $|\varepsilon| \neq 0$ sufficiently small, system (54) has at least 7 limit cycles surround the origin.

\section{Competing Interests}

The author declares that there are no competing interests.

\section{Acknowledgments}

The author was supported by Sichuan Provincial Scientific Research and Innovation Team in Universities (Grant no. 14TD0040), the Natural Science Foundation of Education Department of Sichuan Province (Grant no. 15ZA0337), the 
Major Projects of Aba Teachers University (Grant no. ASA1509), and Provincial Discipline Open Platform Project of Xihua University (szjj2015-060).

\section{References}

[1] D. Hilbert, "Mathematische probleme," in Proceedings of the 2nd International Congress of Mathematics, Nachrichten von der Gesellschaft der Wissenschaften zu Göttingen, MathematischPhysikalische Klasse, pp. 253-297, Paris, France, 1900.

[2] Y. Ye, Theory of Limit Cycle, Shanghai Scientific and Technical, Shanghai, China, 1984.

[3] Y. Ye, Qualitative Theory of Polynomial Differential Systems, Shanghai Scientific and Technical Publishers, Shanghai, China, 1995.

[4] A. Buică and J. Llibre, "Averaging methods for finding periodic orbits via Brouwer degree," Bulletin des Sciences Mathématiques, vol. 128, no. 1, pp. 7-22, 2004.

[5] J. Llibre, D. D. Novaes, and M. A. Teixeira, "On the birth of limit cycles for non-smooth dynamical systems," Bulletin des Sciences Mathématiques, vol. 139, no. 3, pp. 229-244, 2015.

[6] S. Li M, Y. Zhao L, and L. Cen X, "Limit cycles for a class of discontinuous planar quadratic differential system," Scientia Sinica Mathematica, vol. 45, pp. 43-52, 2015.

[7] J. Llibre and A. C. Mereu, "Limit cycles for discontinuous quadratic differential systems with two zones," Journal of Mathematical Analysis and Applications, vol. 413, no. 2, pp. 763-775, 2014.

[8] J. Llibre, B. D. Lopes, and J. R. De Moraes, "Limit cycles for a class of continuous and discontinuous cubic polynomial differential systems," Qualitative Theory of Dynamical Systems, vol. 13, no. 1, pp. 129-148, 2014.

[9] X. Chen and Z. Du, "Limit cycles bifurcate from centers of discontinuous quadratic systems," Computers and Mathematics with Applications, vol. 59, no. 12, pp. 3836-3848, 2010.

[10] R. M. Martins and A. C. Mereu, "Limit cycles in discontinuous classical Liénard equations," Nonlinear Analysis: Real World Applications, vol. 20, pp. 67-73, 2014.

[11] J. Libre and M. A. Teixeira, "Limit cycles for $m$-piecewise discontinuous polynomial Liénard differential equations," Zeitschrift für angewandte Mathematik und Physik, vol. 66, no. 1, pp. 51-66, 2015.

[12] M. Han and L. Sheng, "Bifurcation of limit cycles in piecewise smooth systems via Melnikov function," The Journal of Applied Analysis and Computation, vol. 5, no. 4, pp. 809-815, 2015.

[13] Y. Tian and P. Yu, "Center conditions in a switching Bautin system," Journal of Differential Equations, vol. 259, no. 3, pp. 1203-1226, 2015.

[14] C. Chicone and M. Jacobs, "Bifurcation of limit cycles from quadratic isochrones," Journal of Differential Equations, vol. 91, no. 2, pp. 268-326, 1991.

[15] N. N. Bautin, "On the number of limit cycles which appear with the variation of the coefficients from an equilibrium position of focus or center type," Mathematics of the USSR-Sbornik, vol. 100, pp. 397-413, 1954.

[16] J. Llibre and G. Swirszcz, "On the limit cycles of polynomial vector fields," Dynamics of Continuous, Discrete \& Impulsive Systems. Series A. Mathematical Analysis, vol. 18, pp. 203-214, 2011. 


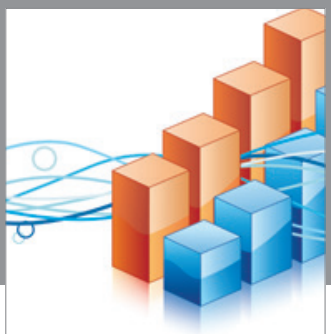

Advances in

Operations Research

vatem alat4

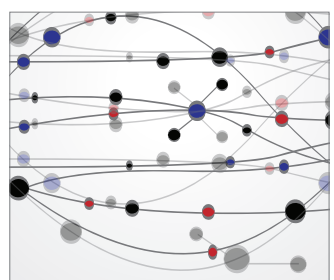

\section{The Scientific} World Journal
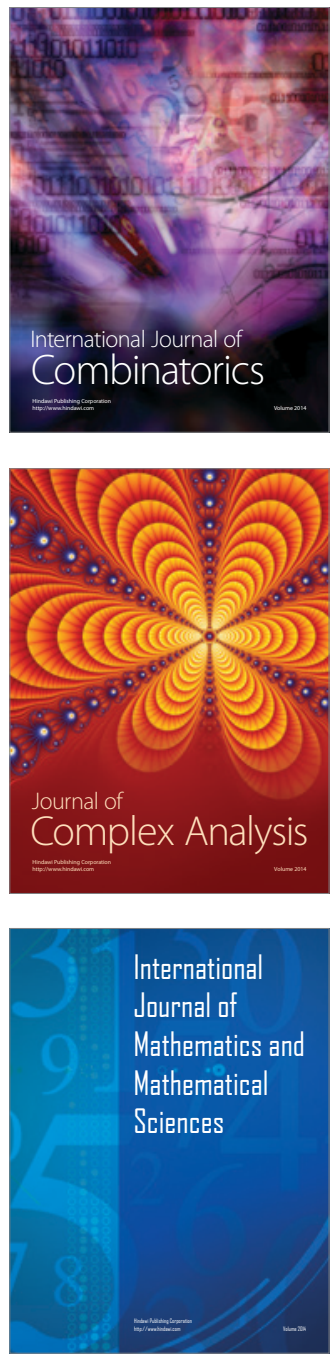
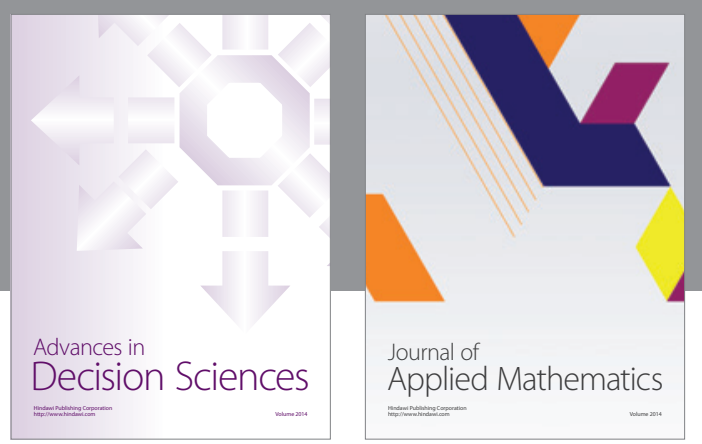

Algebra

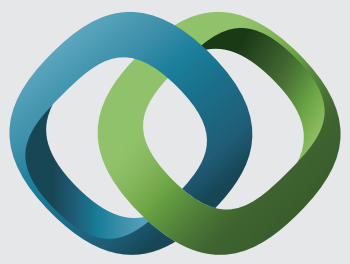

\section{Hindawi}

Submit your manuscripts at

http://www.hindawi.com
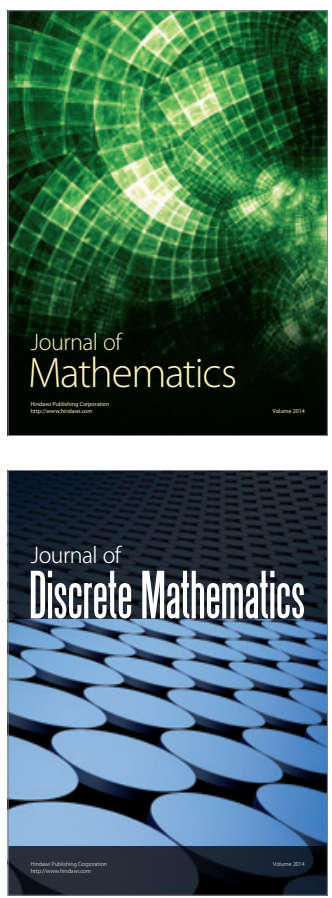

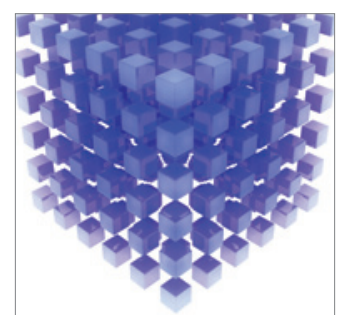

Mathematical Problems in Engineering
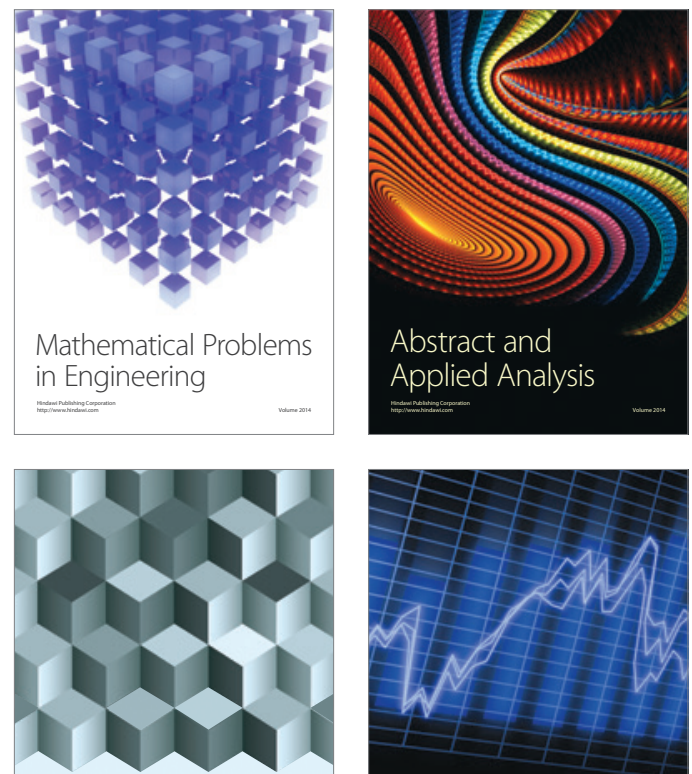

Journal of

Function Spaces

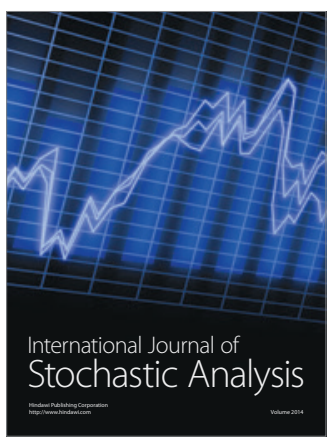

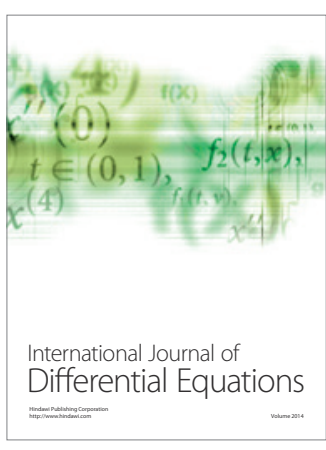
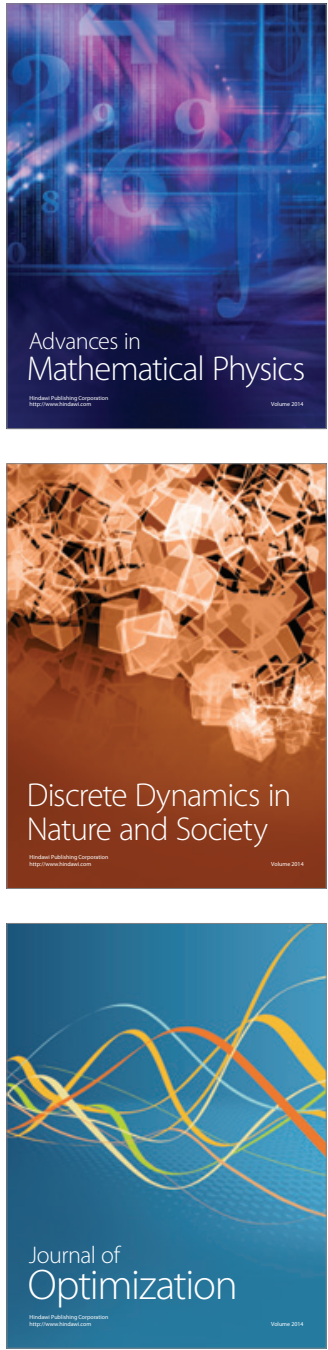